\title{
Faecal glucocorticoid metabolite monitoring as a measure of physiological stress in captive and wild vervet monkeys
}

Christopher Young ${ }^{\mathrm{a}, \mathrm{b}, \mathrm{c}}$, Andre Ganswindt ${ }^{\mathrm{a}}$, Richard McFarland ${ }^{\mathrm{d}, \mathrm{e}}$, Charon de Villiers ${ }^{\mathrm{f}}$, Joritha van Heerden ${ }^{\mathrm{f}}$, Stefanie Ganswindt ${ }^{\mathrm{a}}$, Louise Barrett ${ }^{\mathrm{b}, \mathrm{c}}$, and S. Peter Henzi ${ }^{\mathrm{b}, \mathrm{c}}$

${ }^{a}$ Endocrine Research Laboratory, Department of Anatomy and Physiology, Faculty of Veterinary Science, University of Pretoria, Pretoria, Republic of South Africa.

${ }^{b}$ Applied Behavioural Ecology and Ecosystems Research Unit, University of South Africa, Pretoria, Republic of South Africa.

${ }^{c}$ Department of Psychology, University of Lethbridge, Alberta, Canada.

${ }^{d}$ Department of Anthropology, University of Wisconsin-Madison, Madison, United States.

${ }^{e}$ Brain Function Research Group, School of Physiology, University of the Witwatersrand, Republic of South Africa.

${ }^{f}$ Primate Unit and Delft Animal Centre, South African Medical Research Council, Cape Town, Republic of South Africa.

\section{Corresponding author: Dr Christopher Young}

Email: christopher.young@uleth.ca

\section{Highlights}

- Biological and physiological validation of fGCM monitoring.

- ACTH challenge test was conducted on captive vervet monkeys.

- Cortisol assay is the most appropriate EIA for the species.

- Rank-reversal had no effect on fGCM levels of other group members. 


\begin{abstract}
The development of non-invasive techniques to analyse physiological stress in mammalian species has revolutionised field-based endocrinology. However, careful validation of the methods used to determine faecal glucocorticoid metabolite (fGCM) and other hormone concentrations are required on a species- and sex-specific basis. In this study, we performed an adrenocorticotropic hormone (ACTH) stimulation test on four (two male and two female) captive vervet monkeys (Chlorocebus pygerythrus) to determine the most appropriate enzyme immunoassay (EIA) from a suite of available EIAs. Furthermore, we took advantage of a potentially stressful event in our wild vervet population from Samara Private Game Reserve, South Africa, to examine if an alpha-beta female rank reversal increases the physiological stress of those individuals directly involved, as well as other group members. Both our physiological and biological validation studies revealed that a cortisol assay was the most appropriate EIA for monitoring fGCM alterations in vervet monkeys. In addition, we found that the observed rank-reversal had no significant effect on the physiological stress levels of uninvolved group members. Our study highlights that physiological validation is imperative and, where possible, should be conducted in parallel with a carefully considered biologically-relevant test under natural conditions. Overall, our results provide a necessary step for future studies to examine physiological stress of vervet monkeys via fGCM monitoring by validating a suitable EIA for this species. This paves the way for future research into the health and welfare of both captive and wild vervet monkeys, and will allow researchers to assess the behavioural, social and ecological correlates of physiological stress levels of this species.
\end{abstract}

Keywords: ACTH challenge, adrenocorticotropic hormone test, cortisol, dominance, female rank-reversal, stress validation 


\section{Introduction}

Stress can be defined as the generic term for any stimulus that threatens or appears to threaten the homeostasis of an individual (Selye, 1936; Wielebnowski, 2003). The physiological stress response is an adaptive reaction whereby, under duress, the body exhibits a fight or flight response to mobilise free energy to combat the current stressor (defined as stimuli which is uncontrollable and/or unpredictable; McEwen and Wingfield, 2003; Romero et al., 2009; Sapolsky, 2002). This response leads to a suite of physiological changes to deal with an unknown or uncertain situation including increased heart rate and blood pressure, enhanced cognition and decreased pain perception, as well as the mobilization of energy (McEwen and Wingfield, 2003; Romero et al., 2009; Sapolsky, 2002). An essential component of the response to the stressor is the activation of the hypothalamic-pituitary-adrenocortical (HPA) axis. Activation of the HPA axis results in an elevation in glucocorticoid concentrations, which in turn mobilise energy to both the central nervous system and muscle tissue in order to combat the stressor. This acts to enhance the survival of an individual and, once the uncertainty, diminishes a negative feedback ensues resulting in a decrease in glucocorticoid levels back to baseline via deactivation of the HPA axis (Sapolsky, 2002; Sheriff et al., 2011). Prolonged activation of the HPA axis in response to continuously perceived stressors can, however, have deleterious health consequences (Creel et al., 2013), such as an impaired immune response, reduced reproductive function, and a shorter lifespan (Capitanio, 2011; Cohen et al., 2007; Sapolsky, 2005; Segerstrom and Miller, 2004). Thus, monitoring concentrations of glucocorticoids or its metabolites can be a valuable tool to provide information on the level of physiological stress experienced by animals.

In recent years, non-invasive sample collection techniques have become invaluable tools in modern behavioural ecology to understand stress-related behavioural strategies as well as animal welfare issues (Creel et al., 2013; Higham, 2016). These non-invasive methods have allowed the measurement of several hormone metabolites from faeces, urine and saliva, including glucocorticoid metabolites to measure physiological stress (Behringer and Deschner, 2017; Creel et al., 2013; Higham, 2016; Hodges et al., 2010; McFarland et al., 2013; Young et al., 2014). Historically, peripheral hormone concentrations were determined using blood samples. This method proved to be problematic in the wild, because sample collection usually requires the subjects to be restrained via darting or trapping, a procedure in itself which is perceived as a stressor to the animal, thus rendering the physiological evaluation of their stress levels redundant (Hodges et al., 2010). Capture, restraint and anaesthesia can also potentially have negative and dangerous consequences to an animal's health (Behringer and Deschner, 2017; Hodges et al., 2010).

Glucocorticoids circulating in the blood stream are processed by the liver and excreted via the kidneys in urine or via bile into the gut as glucocorticoid metabolites (McEwen and Wingfield, 2003; Sapolsky, 2002; Sheriff et al., 2011), and examination of these metabolites can therefore be conducted non-invasively via collection and analysis of urine or faeces. Collection of these non-invasive samples allow researchers to analyse hormone metabolites without compromising the sample or health of the individual in both captive and wild conditions (Higham, 2016; Hodges et al., 2010). 
It is important to note that non-invasive physiological stress analyses must be validated carefully to ensure that the measured glucocorticoid metabolites reliably reflect biologically relevant signals (Bahr et al., 2000; Heistermann et al., 2006; Palme et al., 2005). Validation, in this sense, usually refers to artificially activating an animal's HPA axis to induce changes in plasma glucocorticoid levels. Subsequently one must determine which of the available test systems reliably measures related alterations in glucocorticoid metabolite concentrations as an index of adrenocortical function (Bahr et al., 2000; Heistermann et al., 2006; Palme et al., 2005; Sheriff et al., 2011). In this regard, the most widely accepted standard procedure is the adrenocorticotrophic hormone (ACTH) challenge test (Behringer and Deschner, 2017; Heistermann et al., 2006; Sheriff et al., 2011). The metabolism of glucocorticoids can vary between the sexes leading to disparity in the type and concentration of metabolites excreted (Touma et al., 2003). Therefore, it is important that validation should also determine the EIA that maximises metabolite recovery for both males and females (Heistermann et al., 2006; Hodges et al., 2010). Here, we aim to validate measurement of the physiological stress response in both male and female vervet monkeys (Chlorocebus pygerythrus) using the ACTH challenge test (Heistermann et al., 2006). The multifaceted environmental and social stressors which vervet monkeys are exposed to make them a model species in which to investigate the behavioural and physiological adaptations an organism makes in response to frequently changing conditions. Additionally, a valid measure of physiological stress levels via the ACTH challenge will pave the way for research into the welfare of these animals in both captive and wild conditions.

One potential disadvantage of the ACTH challenge test is that it may overstimulate the adrenal gland so that even a less specific assay could detect changes in fGCM output. In addition to using a physiological challenge to determine the reliability of a test system to examine stress steroid metabolite alterations, it has also been suggested to evaluate fGCM output via a biological validation (Sheriff et al., 2011), to confirm that the chosen assay detects biologically relevant changes. To do so, we examined the effect of a potentially stressful event in a group of wild vervet monkeys, specifically, an alpha-beta female rankreversal. Like many cercopithecines, female vervet monkeys are philopatric, and the dominance hierarchy is arranged via matrilines (van Schaik and van Hooff, 1983; Wrangham, 1980). Female philopatry leads to long periods of stability in the female hierarchy, with few periods of social instability (Cavigelli and Caruso, 2015; Cheney and Seyfarth, 2009). In female chacma baboons (Papio ursinus), for example, it has been shown that instability in the female hierarchy can lead to increased stress, especially for those females whose rank positions are particularly at risk (Engh et al., 2006). We use used our observed alpha-beta rank reversal as a biologically-relevant test, which allowed us to examine physiological stress both in the females involved in the reversal, as well as across all adult group members.

Our study therefore had two aims. First, we examined the suitability of five different enzyme immunoassays for monitoring adrenocortical function in vervet monkeys by determining stress-related physiological responses in faeces after performing an ACTH challenge test for both males and females. Second, our aim was to investigate the physiological consequences of a rare social event, a physically aggressive, alpha/beta rank-reversal in the female 
dominance hierarchy, in our wild vervet study group. In doing so, we provided the necessary validation to determine if our chosen assay can detect biologically relevant signals.

\section{Methods}

\subsection{Captive study and ACTH challenge}

We conducted an ACTH challenge test on four captive housed adult vervet monkeys ( 2 males and 2 females) at the Primate Unit and Delft Animal Centre (PUDAC) of the South African Medical Research Council, Cape Town, South Africa. The animals are part of an indoor breeding colony with the room temperature maintained at $23-26{ }^{\circ} \mathrm{C}$, with $45-50 \%$ humidity and a $12 \mathrm{~h}$ photoperiod. For the duration of the study individuals were housed in single cages $(0.9 \mathrm{~m} \times 0.7 \mathrm{~m} \times 1.2 \mathrm{~m})$, which were fitted with full perches, foraging pans and mesh communication panels. Animals were fed three times a day with access to water ad libitum. At the time, none of the individuals in the study had been used in other experimental procedures. All animals were housed and maintained in accordance with the South African National Standard for the Care and Use of Animals for Scientific Purposes (SANS, 10386:2008). The study (Ref: 02/15) was approved by the Ethics Committee for Research on Animals (ECRA) of the South African Medical Research Council and adhered to the laws and guidelines of South Africa.

The ACTH challenge test lasted a total of 7 days. The monkeys were housed in cages three days prior to injection in order to determine baseline levels of glucocorticoid metabolites. Faecal samples were collected during this time from each individual. On day four, each animal was injected intramuscularly with 1-2 IU/kg of Synacthen ${ }^{\circledR} \operatorname{depot}$ (Novartis, South Africa (Pty) Ltd). All produced faecal material was collected for three days post-injection. Samples contaminated with urine were discarded. All samples collected were placed in individually labelled plastic vials, and frozen immediately upon collection at $-20^{\circ} \mathrm{C}$. Faecal samples were then transported on dry ice to the Endocrine Research Laboratory, University of Pretoria for further analysis.

\subsubsection{Wild study and biological validation}

Data for this study were collected on from a wild group of vervet monkeys on the Samara Private Game Reserve, Eastern Cape, South Africa. The group comprised of 29 individuals, including nine adult females and three adult males. Study animals were fully habituated to the presence of researchers and could be individually identified via physical characteristics (Pasternak et al., 2013). Data and faecal material were collected between $1^{\text {st }}$ June and $30^{\text {th }}$ September 2015 for these analyses, but are part of a long-term study in which the monkeys have been observed continuously since 2008 . On the $2^{\text {nd }}$ of July 2015, we observed the beta female win an aggressive encounter for the first time against the alpha female. Such wins up the hierarchy for females are very rare (Young et al., 2017). Over the next three months we witnessed a further 92 dominance interactions between these two females and all but two (i.e., $98 \%$ ) were won by the beta female. 
Ethical note: For the wild vervet study all protocols were non-invasive and adhered to the laws and guidelines of South Africa and Canada. Procedures were approved by the University of Lethbridge Animal Welfare Committee (Protocols 0702 and 1505).

\subsubsection{Behavioural data collection}

Data on agonistic interactions were collected ad libitum as part of our daily data collection protocol for the wild vervet group (McFarland et al., 2015; Pasternak et al., 2013; Young et al., 2017). We recorded all dyadic agonistic contests that occurred in our study group. The loser of a dyadic agonistic bout was determined to be the individual who was last to show submission. Additionally, we recorded spontaneous submission, where an individual would show submissive behaviour without provocation of the partner when the partner was within close proximity $(<2 \mathrm{~m})$. The individual showing spontaneous submission was considered to lose the contest. We calculated dominance hierarchies separately for three consecutive months; for the month before $(\mathrm{N}=57)$, the month directly after $(\mathrm{N}=140)$ and the month starting four weeks after the first observed defeat of the alpha by the beta female $(\mathrm{N}=112)$. An integrated, intersexual hierarchy was constructed as females can out-rank many males in our wild vervet population (Young et al., 2017), and rank was expressed as a standardized normalized David's score (de Vries et al., 2006), using the R package "DomiCalc" (Schmid and de Vries, 2013) in R 2.14.0 (R Development Core Team, 2012). The individual with the highest David's score is the highest ranked individual in the dominance hierarchy. In order to compare between our time periods we standardized the David's scores by dividing all individuals' scores by the highest score obtained. Therefore, the highest ranked individual has a score of 1 (Henzi et al., 2013; Young et al., 2017).

\subsubsection{Faecal sample collection in the wild}

As part of a long-term project investigating vervet monkey social behaviour and its endocrine correlates, two faecal samples were collected from every adult group member each month. For the purposes of this analysis, fortnightly samples from all adult individuals were collected following the date of the first aggressive interaction won by the beta female. This gave a total of 133 samples (mean $=7.75 \pm 1.91$ SD per individual). These samples were collected in the field ad libitum within 15 minutes of defecation. Faecal samples were thoroughly mixed and $2-5 \mathrm{~g}$ transferred in a plastic vial and stored on ice in a thermos, and were then transferred to a $-20^{\circ} \mathrm{C}$ freezer at the end of each field day. Samples were collected throughout the field day, so a sample could be in the thermos from a minimum of less than an hour to a maximum of 10 hours, but the temperature inside the thermos was always below freezing and samples were always frozen when transferred to the freezer at the end of the day. Faecal samples were kept frozen and transported on dry ice to the Endocrine Research Laboratory, University of Pretoria.

\subsection{Faecal sample processing and enzyme immunoassay}

All faecal samples were lyophilised on arrival at the laboratory, and the resulting dried faecal matter pulverized and sieved through a mesh to remove fibrous material (Ganswindt et al., 2010). Between $0.10-0.11 \mathrm{~g}$ of the faecal powder was then extracted. Extraction involved 
vortexing for 15 minutes with $80 \%$ ethanol in water $(3 \mathrm{ml})$. Following centrifugation for 10 minutes at $1500 \mathrm{~g}, 1.5 \mathrm{ml}$ of the respective supernatants was transferred into micro-centrifuge tubes ready for hormone analysis. Extraction efficiency, determined by the recovery of randomly selected spiked faecal samples, was $103.1 \% \pm 14.9 \%$ (mean $\pm \mathrm{SD}, \mathrm{n}=10$ ).

A subset of faecal extracts from the four ACTH treated animals was measured for immunoreactive faecal glucocorticoid metabolite (fGCM) concentrations using 5 different enzyme-immunoassays: (i) an 11-oxoetiocholanolone I (detecting 11,17 dioxoandrostanes; 11,17-DOA), (ii) an 11-oxoetiocholanalone II (detecting fGCMs with a 5 $\beta$-3 $\alpha$-ol-11-one structure; $3 \alpha, 11$-oxo-CM), (iii) a $5 \alpha$-pregnane-3 $\beta, 11 \beta, 21$-triol-20-one (measuring $3 \beta, 11 \beta$ diol-CM), iv) a cortisol, and v) a corticosterone EIA. Detailed assay characteristics, including full descriptions of the assay components and cross-reactivities have been provided by Palme and Möstl (1997) for 11,17-DOA, cortisol and corticosterone, by Möstl et al. (2002) for $3 \alpha, 11$-oxo-CM, and by Touma et al., (2003) for the EIA measuring 3 $\beta, 11 \beta$-diol-CMs. Subsequently, faecal extracts from wild vervet samples were analysed using only the Cortisol EIA. The sensitivities of the EIAs used are $0.6 \mathrm{ng} / \mathrm{g}$ dry weight (DW) (Cortisol, 11oxoetiocholanolone I, and 11-oxoetiocholanolone II EIA), $1.8 \mathrm{ng} / \mathrm{g}$ DW (Corticosterone

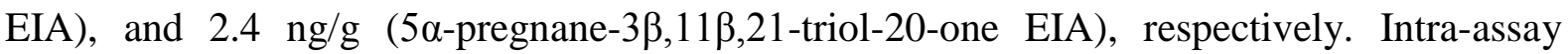
coefficients of variation of high- and low-value quality controls $(\mathrm{n}=16 ; 15$, respectively) were $4.8 \%$ and $5.8 \%$ (Cortisol EIA), 3.1\% and 5.7\% (11-oxoetiocholanolone I EIA), $5.3 \%$ and $5.8 \%$ (11-oxoetiocholanolone II EIA), 5.7\% and 6.0\% (Corticosterone EIA), and $3.8 \%$ and $4.2 \%$ (5 $\alpha$-pregnane-3 $\beta, 11 \beta, 21$-triol-20-one EIA), respectively. Inter-assay coefficients of variation of high- and low-value quality controls $(n=18 ; 25$, respectively) were only determined for the Cortisol EIA: $13.1 \%-15.6 \%$. For the assay of choice (cortisol EIA), serial dilutions of extracted faecal samples $(n=2)$ gave displacement curves, which were parallel to the standard curve (1.7\% and 3.9\% deviation in slope, respectively). Accuracy, determined by the recovery of added standard in low, medium, and high concentrations in steroid extracts $(n=2)$ ranged between $94-108 \%$. All steroid concentrations are given as ng/g faecal dry weight (DW). All EIAs were performed at the Endocrine Research Laboratory, University of Pretoria, as described previously (Ganswindt et al., 2002).

\subsection{Statistical analysis}

Descriptive statistics were used to determine the initial rise, peak and fall in fGCM levels following injection of ACTH. First, baseline levels were calculated on an individual basis using an iterative process described by Brown et al. (1991). For each individual, the mean and standard deviation (SD) were calculated for all samples pre-injection. All fGCM measurements that were greater than 2 SDs above the mean were excluded and the mean was recalculated. This process was repeated until no values exceeded the mean plus 2 SDs. Once each individual's baseline was calculated it was used to determine a significant rise in fGCM levels - this was considered a rise of greater than 100\% from the baseline (Brown et al., 1991). 
In order to perform a biological validation, we used the same method on our wild monkey group for the alpha and beta female during the female rank reversal. We used the samples collected one month prior to the rank change to determine baseline levels and compared this to the peak fGCM level shown by the females after the rank change. To examine the overall effect of a female rank reversal on our wild monkey group, we used a generalised linear mixed model (GLMM; Bolker et al., 2009). Our response variable was individual fGCM levels (ng/g) and the predictor variable was a binomial factor of before or after rank change (i.e., yes/no, $\mathrm{N}=83$ faecal samples). The response variable was $\log$ transformed to achieve normal distribution. We included individual ID as a random effect, specifying both random intercepts and slopes. The alpha and beta female were excluded from this analysis. We ran the GLMM in R 2.14.0 (R Development Core Team, 2012) using the "lme4" package (Bates et al., 2012). The model was fitted with a log-link function, and likelihood ratio tests were calculated using the R function "anova". We used the "MuMIn" package (Barton, 2013) to generate marginal and conditional $\mathrm{R}^{2}$ values for the fixed effect and whole model respectively. All assumptions including checks for influential cases and collinearity were respected for the analysis.

Furthermore, using a loess regression with $\mathrm{r}$ function loess we constructed a graphical representation of the fGCM levels of the alpha and beta females compared to the other group members for the sample collection period. Where appropriate, mean values \pm SD were reported. Following Colquhoun (2014), $\mathrm{P}=0.05,0.01$ and 0.001 was considered to indicate weak, moderate and strong evidence respectively for effects.

\section{Results}

\subsection{ACTH challenge}

For all five EIAs we measured a subset of samples (7-11 samples per individual per assay depending on defecation rate within the 24 hour period on the captive monkeys) from 24 hours before ACTH drug administration to 50 hours after administration. All EIAs detected an increase in fGCM concentrations post-ACTH drug administration (see table 1). For all study subjects the greatest average increase in fGCM levels was revealed when using the cortisol EIA (two males $=214 \%$ and $428 \%$, two females $=303 \%$ and $1033 \%$ increase above baseline, see table 1). We therefore analysed all subsequent samples with this EIA. The peak rise in fGCM metabolites occurred $29.5 \pm 4.37$ hours after injection (mean $\pm \mathrm{SD}$; range 25.58 -33.33 hours). Individuals took an average of $55.75 \pm 13.29$ hours (mean \pm SD; range $43.0-$ 73.8 hours) to return to baseline fGCM levels (figure 1). 
Table 1: The results of the fGCM measurements from the ACTH challenge test using 5 EIAs. Shown are the identities of the four study subjects, their sex, baseline fGCM levels before injection, and their maximum rise and percentage increase in maximum fGCM rise compared to the baseline. The highest percentage increases for each individual were all observed in the cortisol EIA (highlighted in bold).

\begin{tabular}{|c|c|c|c|c|c|c|c|c|c|c|c|c|c|c|c|}
\hline \multirow{3}{*}{ Sex } & \multicolumn{15}{|c|}{ Enzyme immunoassay } \\
\hline & \multicolumn{3}{|c|}{ 11-oxoetiocholanolone I } & \multicolumn{3}{|c|}{ 11-oxoetiocholanolone II } & \multicolumn{3}{|c|}{ Corticosterone } & \multicolumn{3}{|c|}{$\begin{array}{c}5 \alpha \text {-pregnane-3 } \beta, 11 \beta, 21 \text {-triol- } \\
20 \text {-one }\end{array}$} & \multicolumn{3}{|c|}{ Cortisol } \\
\hline & $\begin{array}{c}\text { Baseline } \\
\text { (ng/g) }\end{array}$ & $\begin{array}{l}\text { Max } \\
\text { rise }\end{array}$ & $\begin{array}{l}\text { Percent } \\
\text { increase }\end{array}$ & $\begin{array}{l}\text { Baseline } \\
\text { (ng/g) }\end{array}$ & Max rise & $\begin{array}{l}\text { Percent } \\
\text { increase }\end{array}$ & $\begin{array}{l}\text { Baseline } \\
\text { (ng/g) }\end{array}$ & $\begin{array}{l}\text { Max } \\
\text { rise }\end{array}$ & $\begin{array}{l}\text { Percent } \\
\text { increase }\end{array}$ & $\begin{array}{l}\text { Baseline } \\
\text { (ng/g) }\end{array}$ & $\begin{array}{l}\text { Max } \\
\text { rise }\end{array}$ & $\begin{array}{l}\text { Percent } \\
\text { increase }\end{array}$ & $\begin{array}{l}\text { Baseline } \\
\text { (ng/g) }\end{array}$ & $\begin{array}{l}\text { Max } \\
\text { rise }\end{array}$ & $\begin{array}{l}\text { Percent } \\
\text { increase }\end{array}$ \\
\hline Male 1 & 116.0 & 353.5 & 304.7 & 369.1 & 886.2 & 240.1 & 200.1 & 329.0 & 164.4 & 1060.6 & 3303.9 & 311.5 & 27.6 & 118.0 & 427.5 \\
\hline Male 2 & 162.7 & 288.8 & 177.5 & 353.2 & 735.6 & 208.2 & 314.2 & 180.8 & 57.5 & 1992.3 & 1930.3 & 96.9 & 42.6 & 91.2 & 214.1 \\
\hline Female 1 & 266.0 & 240.7 & 90.5 & 492.2 & 1207.3 & 245.3 & 258.6 & 342.2 & 132.3 & 1334.5 & 1845.9 & 138.3 & 62.1 & 187.9 & 302.7 \\
\hline Female 2 & 162.7 & 748.9 & 460.3 & 353.2 & 3515.4 & 995.2 & 314.2 & 333.5 & 106.1 & 1992.3 & 3735.6 & 187.5 & 95.4 & 985.8 & 1033.3 \\
\hline
\end{tabular}


Figure 1: Vervet monkey fGCM concentrations (ng/g DW) in relation to administration of ACTH (injection time 0). Shown separately are a) males and b) females, each individual is shown as a solid black or red line and the dotted lines show the corresponding baseline levels before ACTH injection. Peak fGCM levels were 33.25 hours after injection. Profiles were created using the cortisol EIA.

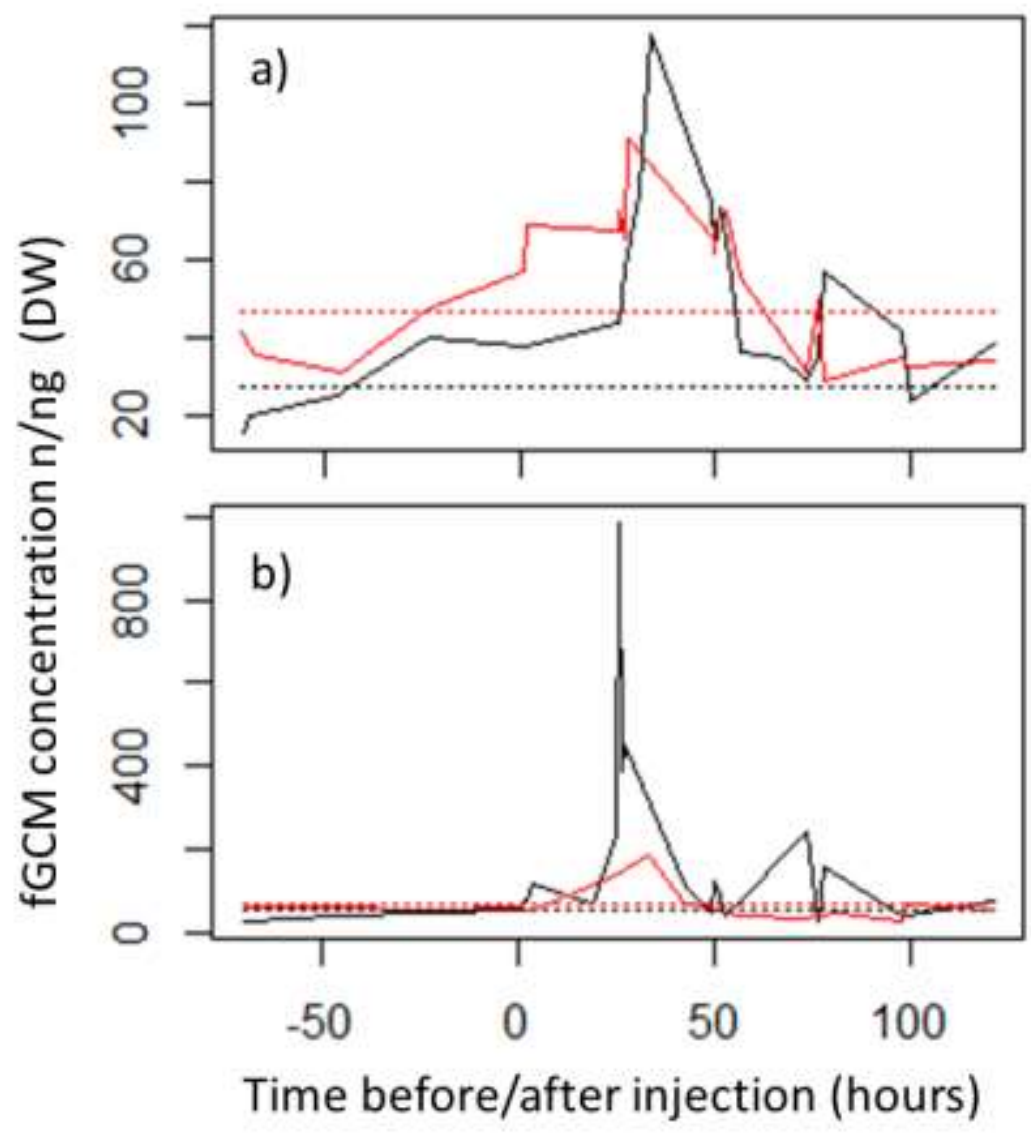

\subsection{Female Rank-reversal}

The first indication of a potential rank change was observed on the $2^{\text {nd }}$ June 2015 when the beta female won a physical aggressive contest against the alpha female. In the following 8 weeks, the beta female won all 51 contests between the two females, with counter-aggression shown in only one of the interactions. For the two months prior to this event, the alpha female had won 15 out of 16 aggressive bouts, with counter-aggression occurring in 2 of these bouts. This shift in the winner of the dyadic contests between the females indicates a clear rank reversal, which was confirmed by the standardized normalized David's Scores (alpha female: before $=1$, after $=0.77$; beta female: before $=0.70$, after $=1,1=$ the alpha position in the hierarchy: see Methods). The old alpha female subsequently maintained the beta position in the hierarchy.

We found that fGCM levels increased above baseline for both the alpha (215\% increase in fGCM levels, mean baseline $=80.1 \mathrm{ng} / \mathrm{g} \mathrm{DW}$, maximum rise $=172.4 \mathrm{ng} / \mathrm{g} \mathrm{DW}$, four days after the event) and beta females (217\% increase in fGCM levels, mean baseline $=54.0 \mathrm{ng} / \mathrm{g}$ $\mathrm{DW}$, maximum rise $=117.2 \mathrm{ng} / \mathrm{g} \mathrm{DW}$, five days after the event) (figure 2). With respect to the other members of the group (i.e., excluding the alpha and beta female), we found no 
effect of the rank reversal on individual's fGCM levels, in fact we found no difference between the null model (only random effects) and that with the binomial fixed effect of before or after rank change (Whole model: $\log$ likelihood $=-446.7, \mathrm{X}^{2}=0.106, \mathrm{df}=1, \mathrm{P}=$ $0.744, R_{\text {MARGINAL }}^{2}=0.001, \mathrm{R}^{2}$ CONDITIONAL $=0.042$, figure 2 ). Therefore, we do not report the result of the fixed effect.

Figure 2: A loess regression of the fGCM concentrations (ng/g DW) of the alpha and beta females (blue) compared to the other group members (red) during the time of the alpha-beta rank change. Day $0=$ the day of the aggressive event that led to the rank change. The shaded areas represent the $95 \%$ confidence intervals.

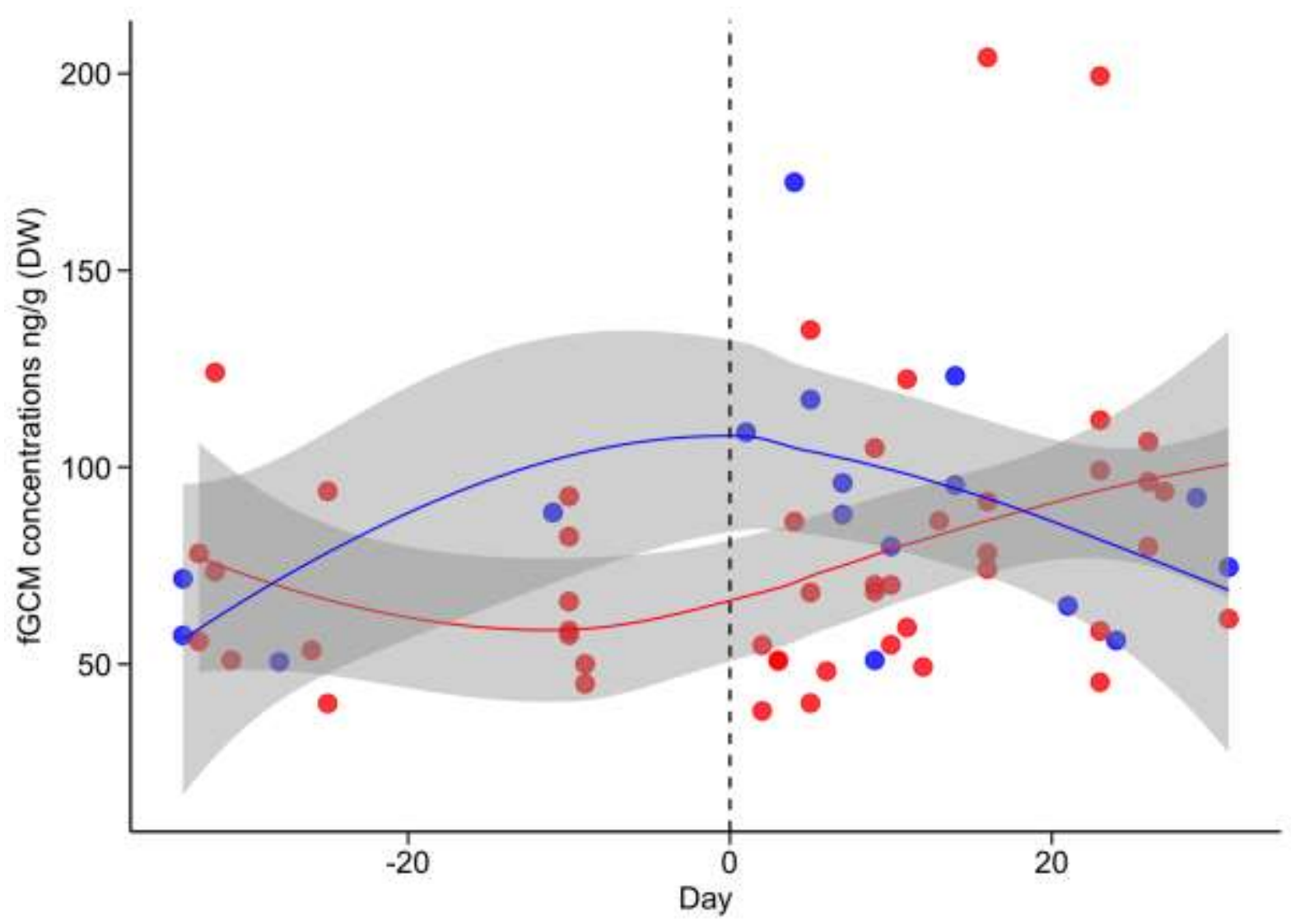

\section{Discussion}

Our results of the ACTH challenge test on the captive housed vervet monkeys confirmed that we are able to reliably monitor alterations in fGCM concentrations using vervet faecal material. The peak in fGCM concentration in our four study subjects occurred between 25 and 33 hours after injection, indicating that vervet monkeys have a time lag from production of GCs in response to an acute stressful event to excretion in the faeces of approximately 24 to 36 hours. This is similar to the results from many other primate species including baboons (Papio cynocephalus cynocephalus, 36.4 hrs: Wasser et al., 1994), long-tailed macaques (Macaca fascicularis, 22 hrs: Bahr et al., 2000), Barbary macaques (Macaca sylvanus, 46 hrs: Heistermann et al., 2006), howler monkeys (Alouatta seniculus, 20.4 hrs: Rimbach et al., 
2013) and chimpanzees (Pan troglodytes, 21-26 hrs: Bahr et al., 2000; Heistermann et al., 2006; for a review see Touma and Palme, 2005). It is worth noting that all assays showed increases in fGCM levels above the $100 \%$ increase threshold, and thus all seem suitable to reliably determine adrenocortical activity in vervet monkeys. However, as native cortisol can be absent or vary greatly in its quantities in faeces (Heistermann et al., 2006), consistency and the level of increase in fGCM levels post-administration was used to identify the most suitable assay of the five EIAs tested. For our vervet monkey study, the cortisol assay showed the greatest increase for both our male and female study subjects (table 1), and therefore this assay was used for further analysis. This result is further supported by our biologicallyrelevant validation in the wild where the alpha and beta female showed similar increases in fGCM levels after the rank change event to that of the physiological challenge test. Combined, these results demonstrated that our cortisol assay is most applicable to examine alterations in fGCM output in both male and female vervet monkeys.

In the wild vervet population, we observed fGCM levels of the alpha and beta female rise above our threshold of a $100 \%$ increase above baseline (217\% and $215 \%$ increase). These elevated levels were determined from faecal samples collected 4 (alpha) and 5 (beta) days after the rank change event, and it is therefore possible that we missed an even more distinct elevation in the period 24-36 hours after the stressor. It is likely that the alpha/beta rank change is a short-term acute stressor and we found no significant difference between the mean fGCM levels during the month prior to the reversal and the two months following the rank change event for any of the individuals in the group, whether directly or indirectly involved in the rank-reversal event. Figure 2 shows a rise in the alpha-beta females towards day 0 and a fall afterwards whereas no similar pattern is observed for the other group members. The figure shows a separation in the bands of the $95 \%$ confidence intervals of the alpha-beta females and the other group members at day 0 , suggesting a clear difference in fGCM levels at this time. It may be that a rank-reversal between two natal females had little effect on the fGCM levels of other group members: from their perspective, their status remained unchanged. Given this, and unlike the disruptive nature of male immigration that is signalled by elevations in physiological stress among baboons (Beehner et al., 2005; Engh et al., 2006), the change of ranks within a natal female vervet hierarchy may not be a dramatic event for other group members. Likewise for the alpha and beta females, although the initial event showed a significant rise in fGCM levels, the acute nature of the stressor shows their levels return to baseline within 7 days of the event (figure 2). During such an uncertain and unpredictable time as the rank-reversal the increased fGCM levels observed are likely related to the acute stress response and activation of the HPA axis to mobilise free energy for the potentially energetically costly rank challenge period (McEwen and Wingfield, 2003; Romero et al., 2009; Sapolsky, 2002). Future long-term data on female vervet monkey rank inheritance and acquisition in combination with frequent individual monitoring of fGCM alterations is required in order to understand fully the physiological consequences of changes in group dynamics and hierarchy instability.

Our results highlight both the need for a direct validation under controlled conditions in order to select the most appropriate assay for the species in question (Bahr et al., 2000; Behringer 
and Deschner, 2017; Heistermann et al., 2006; Hodges et al., 2010) and to validate the assay biologically, ideally in a natural environment. The biological event selected for validation should be chosen carefully given that, in a natural setting, individuals experience many positive and negative social and environmental factors on a daily basis (Cheney and Seyfarth, 2009). These factors are all likely to intermingle and influence the fGCM output of an individual. Although a single event in a natural population may be stressful, it may be extremely difficult to replicate a validation such as that under controlled laboratory conditions. Additionally, what we perceive as a natural stressor for the study subjects may not always be the case from the animals' point of view and the anticipated rise in fGCM levels may not always occur. The logistics of sample collection under natural conditions may also reduce the practicality of a biological test in the wild, as it can be very difficult to collect the required number of samples within the time window needed (ideally all samples produced across a three day window) to assess the impact of a particular event. Generally, non-invasive faecal sample collection provides a useful tool to examine long-term effects of social and environmental factors on physiological stress (Creel et al., 2013; Higham, 2016; Sheriff et al., 2011), but only after careful validation of the methods for the specific species in question.

Overall, we recommend that both physiological (ACTH) and biologically-relevant validation tests should be performed in parallel when attempting to determine the most suitable method to quantify physiological stress levels in mammalian species. A physiological test under controlled conditions of both sexes for the species is vital to determine the most appropriate assay (the "gold standard" being that of the ACTH challenge test: (Behringer and Deschner, 2017) but on its own one cannot determine the biological relevance of the test as the ACTH challenge may overstimulate the adrenal gland. If at all possible, a carefully considered and selected biologically relevant test should also be performed. Likewise, a biological test alone is not recommended, as the uncontrollable nature of natural conditions make for determination of the single stressful event difficult. Due to the species and sex specific nature of fGCMs we recommend using both biological and ACTH challenge tests together, for available EIAs and commercial kits, so that validated results accrue. With the establishment of a reliable non-invasive method (cortisol EIA) for monitoring adrenocortical activity as a measure of fGCM stress levels, we hope our study will stimulate future research examining the various behavioural, ecological, physiological, health and welfare correlates of stress responses among vervet monkeys.

\section{Acknowledgements}

We are grateful to Mark and Sarah Tompkins for permission to work at Samara Private Game Reserve. We are indebted to all students and research assistants that assisted with data collection and to Kitty and Richard Viljoen for logistical support. We are grateful to the PUDAC staff of the SAMRC, Cape Town for their invaluable technical assistance with the captive study and the Endocrine Research Laboratory for their help with the laboratory work at UP. We thank Dr. Mark Sheridan, Dr. James P. Higham and an anonymous reviewer for helpful comments on this manuscript. This research was funded by National Research 
Foundation (South Africa) awards to SPH, Natural Science and Engineering Research Council (Canada) Discovery Grant awards to SPH and LB, the Canada Research Chair program to $\mathrm{LB}$, and a Faculty research grant from the University of the Witwatersrand to RM.

\section{References}

Bahr, N., et al., 2000. Comparative aspects of the metabolism and excetion of cortisol in three individual nonhuman primates. Gen. Comp. Endocrinol. 117, 427-438.

Bartoń, K., 2013. MuMln: multi-model inference, R package version 1.9.13. http://CRAN.Rproject.org/package=MuMIn.

Bates, D., et al., Ime4 - linear mixed effects models using S4 classes. R package version 0.999999-0. http://CRAN.R-project.org/package=Ime4, 2012.

Beehner, J., et al., 2005. The effect of new alpha males on female stress in free-ranging baboons. Anim. Behav. 69, 1211-1221.

Behringer, V., Deschner, T., 2017. Non-invasive monitoring of physiological markers in primates. Horm. Behav.

Bolker, B. M., et al., 2009. Generalized linear mixed models: a practical guide for ecology and evolution. Trends Ecol. Evol. 24, 127-135.

Brown, J., et al., 1991. Impact of season on seminal characteristics and endocrine status of adult free-ranging African buffalo (Syncerus caffer). J. Reprod. Fertil. 92, 47-57.

Capitanio, J. P., 2011. Individual differences in emotionality: social temperament and health. Am. J. Primatol. 73, 507-515.

Cavigelli, S. A., Caruso, M. J., 2015. Sex, social status and physiological stress in primates: the importance of social and glucocorticoid dynamics. Phil. Trans. R. Soc. B. 370, 20140103.

Cheney, D. L., Seyfarth, R. M., 2009. Stress and coping mechanisms in female primates. Adv. Study Behav. Volume 39, 1-44.

Cohen, S., et al., 2007. Psychological stress and disease. JAMA. 298, 1685-1687.

Colquhoun, D., 2014. An investigation of the false discovery rate and the misinterpretation of $p$ values. Open Science. 1, 140216.

Creel, S., et al., 2013. The ecology of stress: effects of the social environment. Funct. Ecol. 27, 66-80.

de Vries, H., et al., 2006. Measuring and testing the steepness of dominance hierarchies. Anim. Behav. 71, 585-592.

Engh, A., et al., 2006. Female hierarchy instability, male immigration and infanticide increase glucocorticoid levels in female chacma baboons. Anim. Behav. 71, 1227- 1237.

Ganswindt, A., et al., 2002. Assessment of testicular endocrine function in captive African elephants by measurement of urinary and fecal androgens. Zoo Biol. 21, 27-36.

Ganswindt, A., et al., 2010. Concentrations of faecal glucocorticoid metabolites in physically injured free-ranging African elephants Loxodonta africana. Wildl. Biol. 16, 323-332.

Heistermann, M., et al., 2006. Comparison of different enzymeimmunoassays for assessment of adrenocortical activity in primates based on fecal analysis. Am. J. Primatol. 68, 257-273.

Henzi, S. P., et al., 2013. Scalar social dynamics in female vervet monkey cohorts. Philos. Trans. R. Soc. Lond. B Biol. Sci. 368, 20120351.

Higham, J. P., 2016. Field endocrinology of nonhuman primates: past, present, and future. Horm. Behav. 84, 145-155.

Hodges, K., et al., 2010. Endocrine monitoring of reproduction and stress. Wild mammals in captivity: principles and techniques for zoo management. 447-468.

McEwen, B. S., Wingfield, J. C., 2003. The concept of allostasis in biology and biomedicine. Horm. Behav. 43, 2-15. 
McFarland, R., et al., 2015. Social integration confers thermal benefits in a gregarious primate. J. Anim. Ecol. 84, 871-878.

McFarland, R., et al., 2013. Physiological stress hormone levels and mating behaviour are negatively correlated in male rhesus macaques (Macaca mulatta). Anim. Biol. 63, 331-341.

Möstl, E., et al., 2002. Measurement of cortisol metabolites in the faeces of ruminants. Vet. Res. Commun. 26, 127-139.

Palme, R., Möstl, E., 1997. Measurement of cortisol metabolites in faeces of sheep as a parameter of cortisol concentration in blood. Zeitschrift für Säugetierkunde- International Journal of Mammalian Biology. 62 192-197.

Palme, R., et al., 2005. Stress hormones in mammals and birds: comparative aspects regarding metabolism, excretion, and noninvasive measurement in fecal samples. Ann. N. Y. Acad. Sci. 1040, 162-171.

Pasternak, G., et al., 2013. Population ecology of vervet monkeys in a high latitude, semi-arid riparian woodland. Koedoe. 55, 01-09.

R Development Core Team, 2012. R: A language and environment for statistical computing. $R$ Foundation for Statistical Computing, Vienna, Austria. http://www.R-project.org/.

Rimbach, R., et al., 2013. Validation of an enzyme immunoassay for assessing adrenocortical activity and evaluation of factors that affect levels of fecal glucocorticoid metabolites in two New World primates. Gen. Comp. Endocrinol. 191, 13-23.

Romero, L. M., et al., 2009. The reactive scope model: a new model integrating homeostasis, allostasis, and stress. Horm. Behav. 55, 375-389.

SANS, 10386:2008. South African National Standard: The Care and Use of Animals for Scientific Purposes. South African National Standard: The Care and Use of Animals for Scientific Purposes, Pretoria, South Africa.

Sapolsky, R., Endocrinology of the stress-response. In: J. Becker, et al., (Eds.), Behavioral Endocrinology. The MIT Press, Cambridge, 2002, pp. 409-450.

Sapolsky, R., 2005. The influence of social hierarchy on primate health. Science. 308, 648-652.

Schmid, V. S., de Vries, H., 2013. Finding a dominance order most consistent with a linear hierarchy: an improved algorithm for the I\&SI method. Anim. Behav. 86, 1097-1105.

Segerstrom, S. C., Miller, G. E., 2004. Psychological stress and the human immune system: a metaanalytic study of 30 years of inquiry. Psychol. Bull. 130, 601.

Selye, H., 1936. A syndrome produced by diverse nocuous agents. Nature. 138, 32.

Sheriff, M. J., et al., 2011. Measuring stress in wildlife: techniques for quantifying glucocorticoids. Oecologia. 166, 869-887.

Touma, C., Palme, R., 2005. Measuring fecal glucocorticoid metabolites in mammals and birds: the importance of validation. Ann. N. Y. Acad. Sci. 1046, 54-74.

Touma, C., et al., 2003. Effects of sex and time of day on metabolism and excretion of corticosterone in urine and feces of mice. Gen. Comp. Endocrinol. 130, 267-278.

van Schaik, C. P., van Hooff, J. A. R. A. M., 1983. On the ultimate causes of primate social systems. Behaviour. 85, 91-117.

Wasser, S., et al., 1994. Excretion rates and metabolites of oestradiol and progesterone in baboon (Papio cynocephalus cynocephalus) faeces. J. Reprod. Fertil. 101, 213-220.

Wielebnowski, N., 2003. Stress and distress: evaluating their impact for the well-being of zoo animals. J. Am. Vet. Med. Assoc. 223, 973-977.

Wrangham, R. W., 1980. An ecological model of female-bonded primate groups. Behaviour. 75, $262-$ 300.

Young, C., et al., 2014. Responses to social and environmental stress are attenuated by strong male bonds in wild macaques. Proc. Natl. Acad. Sci.

Young, C., et al., 2017. Formidable females and the power trajectories of socially integrated male vervet monkeys. Anim. Behav. 125, 61-67. 